\title{
Electromagnetic Scattering by Buried Objects of Low Contrast
}

\author{
DAVID A. HILL, FELLOW, IEEE
}

\begin{abstract}
We use the Born approximation to derive the plane-wave scattering matrix for objects of low dielectric contrast. For general shapes a numerical integration over the volume of the scatterer is required, but analytical expressions are derived for a sphere, a circular cylinder, and a rectangular box (parallelepiped). The plane-wave scattering-matrix theory is used to account for the air-earth interface. $\mathrm{Nu}$ merical results are presented for the scattered near field and far field for plane-wave excitation. The scattered fields are weak for low-contrast objects, but the near-field results have application to electromagnetic detection of buried objects.
\end{abstract}

\section{INTRODUCTION}

$\mathrm{T}$ HE ANALYSIS of electromagnetic scattering by buried objects [1]-[4] is complicated by the presence of the air-earth interface. The plane-wave scattering-matrix formulation [5] provides a convenient means for combining the source, scattering, and interface effects and for including interactions. However, it requires knowing the full plane-wave scattering matrix [6] for the buried object for all incident and scattered plane waves. For scatterers of arbitrary shape where numerical methods are generally required to determine the scattering characteristics, this requires a large amount of computation.

In this paper we use the Born approximation (also called the Rayleigh-Gans approximation [7]) to obtain an approximate expression for the scattering matrix of the buried object in terms of an integral over the volume of the object. For simple shapes (such as a sphere, a circular cylinder, or a rectangular box), the volume integral can be evaluated analytically to obtain a closed-form expression for the scattering matrix. The Born approximation requires small contrast between the electrical properties of the buried object and the earth. This requirement is fairly restrictive, but the low-contrast case is important [8] because it represents a difficult detection situation. For example, the detection of plastic mines in dry, sandy soil is difficult because of the low dielectric contrast [9].

The organization of this paper is as follows. In Section II we review the plane-wave scattering-matrix formulation for the geometry of a buried scatterer [5]. In Section III we use the Born approximation to derive the plane-

Manscript received March 3, 1987; revised September 11, 1987. This work was supported by the U.S. Army Belvoir Research \& Development Center.

The author is with the Electromagnetic Fields Division, National Bureau of Standards, U.S. Department of Commerce, Boulder, CO 80303.

IEEE Log Number 8718450 wave scattering matrix for a buried object of low contrast. Specific results are given for a sphere, a circular cylinder, and a rectangular box. In Section IV we present numerical results for the scattered field above the earth when the incident field is a plane wave. Conclusions and recommendations for further work are contained in Section V.

\section{Plane-Wave Scattering-Matrix Formulation}

The geometry for a scatterer located in a half space is shown in Fig. 1. The unprimed coordinate system $(x, y$, $z$ ) has its origin at the interface $(z=0)$, and the primed coordinate system $\left(x^{\prime}, y^{\prime}, z^{\prime}\right)$ is centered at the scatterer. The region $z<0$ has permittivity $\epsilon$ and permeability $\mu$, and the region $z>0$ has permittivity $\epsilon^{\prime}$ and permeability $\mu^{\prime}$. Both regions are isotropic, but $\epsilon$ and $\epsilon^{\prime}$ can be complex to allow for loss. The time dependence is $\exp (-i \omega t)$.

The more general problem, which includes the source antenna, has been formulated in [5]. In this section we extract only those results from [5] that are needed to analyze the scattering problem. Throughout this paper we follow the notation of Kerns [6].

\section{A. Plane-Wave Representations}

We define region 1 to be a homogeneous half space, $z$ $<0$. In this region we can write the electric field $\boldsymbol{E}_{1}$ as an integral of the plane-wave spectrum

$$
\begin{aligned}
\boldsymbol{E}_{1}(\boldsymbol{r})= & \int_{-\infty}^{\infty} \int_{m=1}^{2} \sum_{m}\left[b_{1}(m, \boldsymbol{K}) \boldsymbol{E}_{m}^{+}(\boldsymbol{K}, \boldsymbol{r})\right. \\
& \left.+a_{1}(m, \boldsymbol{K}) \boldsymbol{E}_{m}^{-}(\boldsymbol{K}, \boldsymbol{r})\right] d k_{x} d k_{y}
\end{aligned}
$$

where $a_{1}$ and $b_{1}$ are plane-wave coefficients, and $r$ is the position vector. A similar expression can be written for the magnetic field [5]. The integrations are taken over real values of the wavenumbers $k_{x}$ and $k_{y} . \boldsymbol{K}$ is given by $\boldsymbol{K}=$ $k_{x} e_{x}+k_{y} e_{y}$, where $e_{x}, e_{y}$, and $e_{z}$ are unit vectors.

The plane-wave field $\boldsymbol{E}_{m}^{ \pm}$is transverse magnetic (TM) for $m=1$ or transverse electric (TE) for $m=2$. The propagation vector $\boldsymbol{k}^{ \pm}$can be written in the following form:

$$
\boldsymbol{k}^{ \pm}=\boldsymbol{K} \pm \gamma \boldsymbol{e}_{z}
$$

where $\gamma=\left(k^{2}-K^{2}\right)^{1 / 2}, k^{2}=\omega^{2} \mu \epsilon$, and $K^{2}=K \cdot K$.

The sign of the square root is chosen so that the imaginary part of $\gamma$ is positive. If the imaginary part of $\gamma$ is 


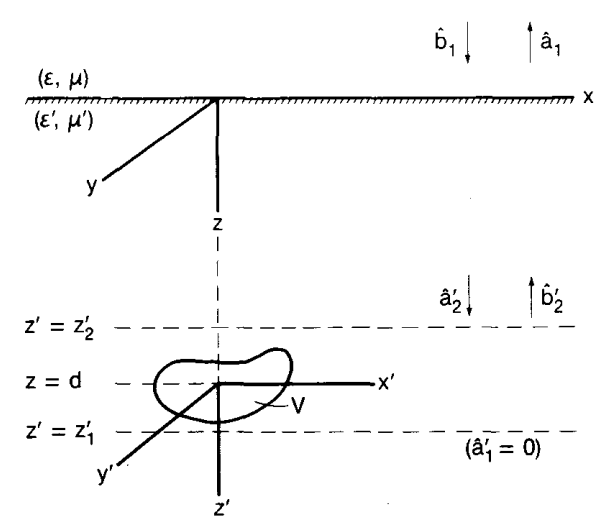

Fig. 1. Geometry for a scatterer located in a half space.

zero, then the sign is chosen so that $\gamma$ is positive real. We define the following transverse unit vectors:

$$
\boldsymbol{\kappa}_{1}=\boldsymbol{K} / K \text { and } \boldsymbol{\kappa}_{2}=\boldsymbol{e}_{z} x \boldsymbol{\kappa}_{1}
$$

which are respectively in and perpendicular to the plane of $\boldsymbol{k}^{ \pm}$and $e_{z}$. The TM plane-wave field is given by

$$
\boldsymbol{E}_{1}^{ \pm}=\left(\boldsymbol{\kappa}_{1} \mp K \gamma^{-1} \boldsymbol{e}_{z}\right) u^{ \pm}
$$

where $u^{ \pm}=\exp \left(i k^{ \pm} \cdot r\right) /(2 \pi)$. The superscript \pm refers to propagation in the $\pm z$ direction. The TE planewave field is given by

$$
\boldsymbol{E}_{2}^{ \pm}=\kappa_{2} u^{ \pm} \text {. }
$$

In some cases, only the transverse components of the electrical field are required. From (1)-(5), we can write

$$
\begin{aligned}
\boldsymbol{E}_{1 t}(\boldsymbol{r})= & \frac{1}{2 \pi} \int \sum_{m}\left[b_{1}(m, \boldsymbol{K}) e^{i \gamma z}\right. \\
& \left.+a_{1}(m, \boldsymbol{K}) e^{-i \gamma z}\right] \boldsymbol{\kappa}_{m} e^{i \boldsymbol{K} \cdot \boldsymbol{R}} d \boldsymbol{K}
\end{aligned}
$$

where $\boldsymbol{r}=\boldsymbol{R}+z \boldsymbol{e}_{z}$ and subscript $t$ denotes the transverse components. When $z$ components are desired, they can be obtained from (1) or from the transverse components and Maxwell's equations. In (6) and throughout the rest of this paper, summation over the values 1 and 2 of the polarization index $m$ and integration over the infinite $k_{x}, k_{y}$ plane are understood.

We define region 2 to be a slab region $\left(-d<z^{\prime}<\right.$ $\left.z_{2}^{\prime}\right)$ between the scatterer and the interface. In this region we can write the transverse electric field in a manner analogous to (6)

$$
\begin{aligned}
\boldsymbol{E}_{2 t}^{\prime}\left(\boldsymbol{r}^{\prime}\right)= & \frac{1}{2 \pi} \int \sum_{m}\left[b_{2}^{\prime}(m, \boldsymbol{K}) \exp \left(-i \gamma^{\prime} z^{\prime}\right)\right. \\
& \left.+a_{2}^{\prime}(m, \boldsymbol{K}) \exp \left(i \gamma^{\prime} z^{\prime}\right)\right] \boldsymbol{\kappa}_{m} \\
& \cdot \exp \left(i \boldsymbol{K} \cdot \boldsymbol{R}^{\prime}\right) d \boldsymbol{K}
\end{aligned}
$$

where $\boldsymbol{r}^{\prime}=\boldsymbol{R}^{\prime}+z^{\prime} \boldsymbol{e}_{z}, \gamma^{\prime}=\left(k^{\prime 2}-K^{2}\right)^{1 / 2}$, and $k^{\prime 2}=$ $\omega^{2} \mu^{\prime} \epsilon^{\prime}$. We could write a similar expression for the field in the region, $z^{\prime}>z_{1}^{\prime}$, but this region contains no sources and does not affect the scattering.

\section{B. Scattering Matrix}

The plane-wave scattering matrix $S_{22}^{\prime}$ of the buried object relates the coefficients of the incoming and outgoing plane waves. The outgoing plane wave coefficients $b_{2}^{\prime}$ can be written [6]

$$
b_{2}^{\prime}(m, K)=\int_{L} \sum_{n} S_{22}^{\prime}(m, K ; n, L) a_{2}^{\prime}(n, L) d L
$$

where $n$ and $\boldsymbol{L}$ represent the polarization and transverse wavenumbers of the incoming plane waves. We could write a similar expression for the coefficients $b_{1}^{\prime}$ of the plane waves scattered in the positive $z^{\prime}$ direction, but we are not concerned with the fields in the region, $z^{\prime}>z_{1}^{\prime}$. For general scatterers, the calculation of $S_{22}^{\prime}$ is a big job. However, for objects of low contrast the Born approximation simplifies the calculations, as shown in Section III.

For later work it is convenient to rewrite (8) in the following shorthand notation [6]:

$$
\hat{b}_{2}^{\prime}=\hat{S}_{22}^{\prime} \hat{a}_{2}^{\prime} \text {. }
$$

Here $\hat{S}_{22}^{\prime}$ is a square matrix representing $S_{22}^{\prime}(m, K ; n, L)$ according to the values of $m, n, K$, and $L$. Similarly, $\hat{b}_{2}^{\prime}$ and $\hat{a}_{2}^{\prime}$ are column matrices representing $b_{2}^{\prime}(m, K)$ and $a_{2}^{\prime}(n, L)$.

\section{Scattered Field in Region 1}

For detection of buried objects, we are interested primarily in the scattered fields in region 1 that is normally free space. In (1), $b_{1}$ is the coefficient of the incident plane waves, and $a_{1}$ is the coefficient of the scattered plane waves. Thus, we need to determine $a_{1}$ in terms of $b_{1}$. This can be done by matching the fields at the interface $(z=$ 0 ) and using the scattering matrix relationship in (8) or (9). The result in shorthand notation is [5]

$$
\hat{a}_{1}=\hat{R}_{11}^{t} \hat{b}_{1}+\hat{T}_{12}^{t} \hat{S}_{22}^{\prime}\left(\hat{1}-\hat{R}_{22}^{t} \hat{S}_{22}^{\prime}\right)^{-1} \hat{T}_{21}^{t} \hat{b}_{1} .
$$

Here $\hat{1}$ is the identity matrix, $\hat{R}_{11}^{t}$ and $\hat{R}_{22}^{t}$ are diagonal reflection-coefficient matrices, $\hat{T}_{12}^{t}$ and $\hat{T}_{21}^{t}$ are diagonal transmission-coefficient matrices, and the superscript $t$ indicates translation. The expressions for the diagonal elements are [5]

$$
\begin{aligned}
& R_{11}^{t}=R_{11}, \quad R_{22}^{t}=R_{22} e^{i 2 \gamma^{\prime} d} \\
& T_{12}^{t}=T_{12} e^{i \gamma^{\prime} d}, \quad T_{21}^{t}=T_{21} e^{i \gamma^{\prime} d}
\end{aligned}
$$

where

$$
\begin{aligned}
& R_{11}=\frac{\eta_{m}-\eta_{m}^{\prime}}{\eta_{m}+\eta_{m}^{\prime}}, \quad R_{22}=\frac{\eta_{m}^{\prime}-\eta_{m}}{\eta_{m}^{\prime}+\eta_{m}} \\
& T_{12}=\frac{2 \eta_{m}^{\prime}}{\eta_{m}+\eta_{m}^{\prime}}, \quad T_{21}=\frac{2 \eta_{m}}{\eta_{m}+\eta_{m}^{\prime}} .
\end{aligned}
$$

The quantities in (12) are plane-wave reflection and transmission coefficients, and the admittances are given by

$$
\begin{aligned}
& \eta_{1}=\omega \epsilon / \gamma, \quad \eta_{2}=\gamma /(\omega \mu) \\
& \eta_{1}^{\prime}=\omega \epsilon^{\prime} / \gamma^{\prime}, \quad \eta_{2}^{\prime}=\gamma^{\prime} /\left(\omega \mu^{\prime}\right) .
\end{aligned}
$$


The physical interpretation of (10) is clear. The first term represents specular reflection $\left(\hat{R}_{11}^{t}\right)$ of the incident field $\left(\hat{b}_{1}\right)$ from the planar interface. The second term is caused by the buried object. It involves transmission into the lower medium $\left(\hat{T}_{21}^{t}\right)$, scattering by the buried object $\left(\hat{S}_{22}^{\prime}\right)$, and transmission back into region $1\left(\hat{T}_{12}^{t}\right)$. The inverse factor represents multiple reflections between the buried object and the interface. The multiple reflections can be shown explicitly if we expand the inverse in a geometric series

$$
\begin{aligned}
\left(\hat{1}-\hat{R}_{22}^{t} \hat{S}_{22}^{\prime}\right)^{-1}= & \hat{1}+\hat{R}_{22}^{t} \hat{S}_{22}^{\prime} \\
& +\left(\hat{R}_{22}^{t} \hat{S}_{22}^{\prime}\right)^{2}+\cdots .
\end{aligned}
$$

If we have weak scattering as in the low-contrast case, then multiple reflections can be neglected, and the first term in (14) is sufficient. In this case, (10) reduces to

$$
\hat{a}_{1} \approx \hat{R}_{11}^{t} \hat{b}_{1}+\hat{T}_{12}^{t} \hat{S}_{22}^{\prime} \hat{T}_{21}^{t} \hat{b}_{1}
$$

\section{BORn ApProximation}

The geometry for a homogeneous scatterer of permittivity $\epsilon_{s}$ imbedded in a homogeneous medium of permittivity $\epsilon^{\prime}$ is shown in Fig. 2. We assume no magnetic contrast so that both media have magnetic permeability $\mu^{\prime}$. The refractive index $n$ of the scatterer is

$$
n=\left(\epsilon_{s} / \epsilon^{\prime}\right)^{1 / 2}
$$

and $n$ can be complex because both $\epsilon^{\prime}$ and $\epsilon_{s}$ can be complex. The Born approximation is valid under the following conditions [7]:

$$
|n-1| \ll 1 \text { and } s\left|k^{\prime}(n-1)\right| \ll 1
$$

where $k^{\prime}=\omega\left(\mu^{\prime} \epsilon^{\prime}\right)^{1 / 2}$ and $s$ is the maximum linear dimension of the scatterer. (The Born approximation is also called the Rayleigh-Gans approximation [7], but we will use Born in this paper to avoid confusion with Rayleigh scattering discussed in Appendix B.) Under the above conditions, the electric field within the scatterer is equal to the incident field $\boldsymbol{E}^{i}$. The scattered field can be computed as the field radiated by the equivalent current density $J_{e}$ located within the scatterer volume

$$
J_{e}\left(r_{s}\right)=-i \omega \epsilon^{\prime}\left(n^{2}-1\right) E^{i}\left(r_{s}\right) .
$$

In this section we will use (18) to derive the scattering matrix for scatterers of low contrast.

\section{A. Scatterers of Arbitrary Shape}

Although we require the scattering matrix $S_{22}^{\prime}$ as defined in (8), we find it easier to derive the dyadic scattering matrix $s_{22}^{\prime}$ first. The two forms contain the same information, and the derivation of $S_{22}^{\prime}$ in terms of the dyadic scattering matrix is given in Appendix A. The dyadic scattering matrix relates the vector coefficients of the incoming and outgoing plane waves as follows [6]:

$$
b_{2}^{\prime}(K)=\int s_{22}^{\prime}(K, L) \cdot a_{2}^{\prime}(L) d L
$$

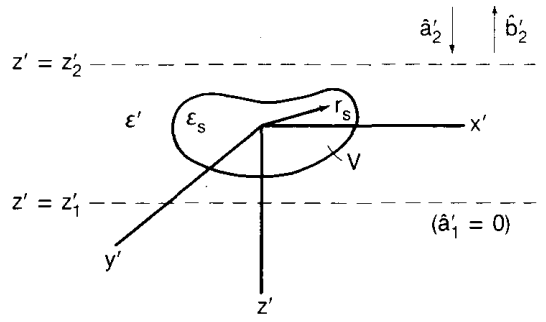

Fig. 2. Geometry for a scatterer of permittivity $\epsilon_{s}$.

The vector plane-wave coefficients are related to the scalar coefficients by

$$
\begin{aligned}
& \boldsymbol{b}_{2}^{\prime}(\boldsymbol{K})=b_{2}^{\prime}(1, \boldsymbol{K})\left(\boldsymbol{\kappa}_{1}+K \gamma^{\prime-1} \boldsymbol{e}_{z}\right)+b_{2}^{\prime}(2, \boldsymbol{K}) \boldsymbol{\kappa}_{2} \\
& \boldsymbol{a}_{2}^{\prime}(L)=a_{2}^{\prime}(1, L)\left(\boldsymbol{\kappa}_{L 1}-L{\gamma_{L}^{\prime}}^{-1} \boldsymbol{e}_{z}\right)+a_{2}^{\prime}(2, L) \boldsymbol{\kappa}_{L 2} .
\end{aligned}
$$

The subscript $L$ on $\gamma^{\prime}$ and $\kappa$ refers to an incoming plane wave with transverse wavenumber $\boldsymbol{L}$.

Kerns [6, pp. 124-129] has presented a general formalism for the scattering matrix, and we follow his approach and notation in our application of the Born approximation. We assume that the incident field is a plane wave of the form

$$
\begin{aligned}
\boldsymbol{E}^{i}\left(\boldsymbol{r}^{\prime}\right)=\frac{\boldsymbol{a}_{2}^{\prime}(\boldsymbol{L})}{2 \pi} \exp \left(i \boldsymbol{l}^{+} \cdot \boldsymbol{r}^{\prime}\right), \\
\text { where } \boldsymbol{l}^{++}=\boldsymbol{L}+\gamma_{L}^{\prime} \boldsymbol{e}_{z} .
\end{aligned}
$$

Then the integral in (19) reduces to a dot product [6]

$$
b_{2}^{\prime}(K)=s_{22}^{\prime}(K, L) \cdot a_{2}^{\prime}(L) .
$$

If we substitute (21) into (18), the equivalent current density of the scatterer is

$$
J_{e}\left(r_{s}\right)=\frac{-i \omega \epsilon^{\prime}\left(n^{2}-1\right) a_{2}^{\prime}(L)}{2 \pi} \exp \left(i l^{+} \cdot r_{s}\right)
$$

The task now is to derive an expression for $\boldsymbol{b}_{2}^{\prime}$ in terms of $J_{e}$ so that we can identify the form of $s_{22}^{\prime}$. Kerns [6, pp. 124-127] has already derived the plane-wave spectrum of the fields radiated by prescribed currents in a volume. If we substitute (23) into his result and perform some algebra, we obtain

$$
\boldsymbol{b}_{2}^{\prime}(\boldsymbol{K})=\frac{i k^{\prime 2}\left(n^{2}-1\right) I_{V}}{8 \pi^{2} \gamma^{\prime}} \pi\left(\boldsymbol{k}^{\prime-}\right) \cdot \boldsymbol{\pi}\left(\boldsymbol{l}^{+}\right) \cdot \boldsymbol{a}_{2}(\boldsymbol{L})
$$

where

$$
I_{V}=\int_{V} \exp \left[i\left(\boldsymbol{l}^{+}-\boldsymbol{k}^{\prime-}\right) \cdot \boldsymbol{r}_{s}\right] d \boldsymbol{r}_{s}
$$

and $V$ is the volume of the scatterer. The projection operator $\pi$ in (24) is defined as [6]

$$
\pi(k)=1-k k / k^{2}
$$


where 1 is the unit dyad. From (22) and (24), we can identify the dyadic scattering matrix

$$
\boldsymbol{s}_{22}^{\prime}(K, L)=\frac{i k^{\prime 2}\left(n^{2}-1\right) I_{V}}{8 \pi^{2} \gamma^{\prime}} \pi\left(\boldsymbol{k}^{\prime-}\right) \cdot \pi\left(l^{++}\right) .
$$

The equivalent scalar form $S_{22}^{\prime}$ is given in Appendix A.

The form of the dyadic scattering matrix for low-contrast scatterers is similar to that for a small dielectric sphere [6], and analytical and numerical comparisons are given in Appendix B. All of the shape dependence is contained in $I_{V}$, and $I_{V}$ is evaluated for some specific shapes in the next section. For very small scatterers, $I_{V}$ is approximately equal to the volume $V$, and $s_{22}^{\prime}$ is proportional to $\left(n^{2}-1\right) V$. This simple dependence is consistent with measurements of the scattered field (and detectability) of plastic mines buried in dry sandy soil [9].

\section{B. Scatterers of Simple Shape}

In general, $I_{V}$ as given by (24) must be evaluated numerically. However, for some simple shapes the integration can be performed analytically. In this section we evaluate $I_{V}$ for a rectangular box (parallelepiped), a circular cylinder, and a sphere. We first rewrite $I_{V}$ in the following equivalent form:

$$
I_{V}=\int_{V} \exp \left(i \boldsymbol{p} \cdot \boldsymbol{r}_{s}\right) d \boldsymbol{r}_{s}
$$

where

$$
\boldsymbol{p}=\boldsymbol{l}^{+}-\boldsymbol{k}^{\prime-}=p_{x} \boldsymbol{e}_{x}+p_{y} \boldsymbol{e}_{y}+p_{z} \boldsymbol{e}_{z} .
$$

Both $p_{x}$ and $p_{y}$ are real, but $p_{z}$ can be complex because $\gamma_{L}^{\prime}$ and $\gamma^{\prime}$ can be complex.

The geometry for a rectangular box of dimensions $2 \mathrm{a}$ by $2 b$ by $2 c$ is shown in Fig. 3(a). For this shape, (27) can be rewritten as

$$
\begin{aligned}
I_{V}= & \int_{-c}^{c} \int_{-b}^{b} \int_{-a}^{a} \exp \left[i\left(p_{x} x^{\prime}+p_{y} y^{\prime}+p_{z} z^{\prime}\right)\right] \\
& \cdot d x^{\prime} d y^{\prime} d z^{\prime} .
\end{aligned}
$$

The integrations in (28) are easily performed to yield

$$
\begin{gathered}
I_{V}=V \frac{\sin \left(p_{x} a\right)}{p_{x} a} \frac{\sin \left(p_{y} b\right)}{p_{y} b} \frac{\sin \left(p_{z} c\right)}{p_{z} c}, \\
\text { where } V=8 a b c .
\end{gathered}
$$

For an electrically small box, all of the sine arguments are small, and $I_{V}$ approaches $V$.

The geometry for a circular cylinder of height $2 h$ and radius $a_{c}$ is shown in Fig. 3(b). For this shape, (27) can be rewritten as

$$
\begin{aligned}
I_{V}= & \int_{-h}^{h} \int_{0}^{2 \pi} \int_{0}^{a_{c}} \exp \left\{i \left[\rho ^ { \prime } \left(p_{x} \cos \phi^{\prime}\right.\right.\right. \\
& \left.\left.\left.+p_{y} \sin \phi^{\prime}\right)+p_{z} z^{\prime}\right]\right\} \rho^{\prime} d \rho^{\prime} d \phi^{\prime} d z^{\prime} .
\end{aligned}
$$

The $z^{\prime}$ integration can be performed to yield a sine function, and the $\phi^{\prime}$ integration can be performed to yield a

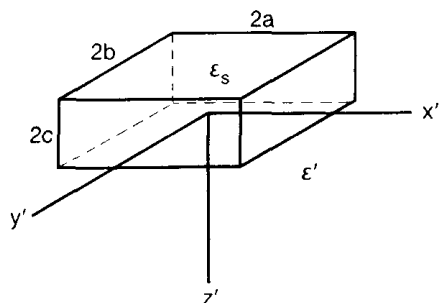

(a)

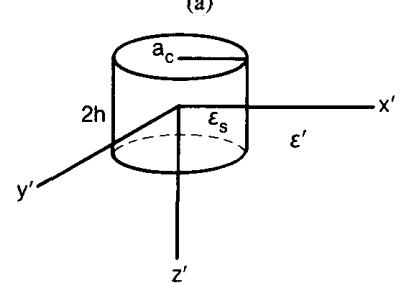

(b)

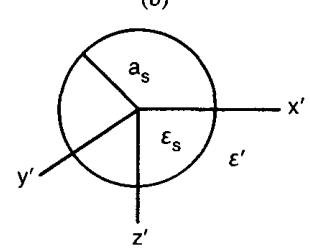

(c)

Fig. 3. Geometry for scatterers of special shape centered at the origin: (a) rectangular box (parallelepiped), (b) circular cylinder, (c) sphere.

zero-order Bessel function [10]

$$
\begin{gathered}
I_{V}=4 \pi h \frac{\sin \left(p_{z} h\right)}{\left(p_{z} h\right)} \int_{0}^{a_{c}} J_{0}\left(u \rho^{\prime}\right) \rho^{\prime} d \rho^{\prime}, \\
\text { where } u=\left(p_{x}^{2}+p_{y}^{2}\right)^{1 / 2} .
\end{gathered}
$$

The $\rho^{\prime}$ integration can be performed by using recurrence relations [10] to yield a first-order Bessel function

$$
I_{V}=V \frac{J_{1}\left(u a_{c}\right)}{\left(u a_{c} / 2\right)} \frac{\sin \left(p_{z} h\right)}{\left(p_{z} h\right)}, \quad \text { where } V=2 h \pi a_{c}^{2} .
$$

For an electrically small cylinder, the arguments of the Bessel function and sine are small, and $I_{V}$ approaches $V$.

The geometry for a sphere of radius $a_{s}$ is shown in Fig. 3(c). For this geometry, (27) can be written in spherical coordinates

$$
\begin{aligned}
I_{V}= & \int_{0}^{a_{s}} \int_{0}^{2 \pi} \int_{0}^{\pi} \exp \left\{i r ^ { \prime } \left[\operatorname { s i n } \theta ^ { \prime } \left(p_{x} \cos \phi^{\prime}\right.\right.\right. \\
& \left.\left.\left.+p_{y} \sin \phi^{\prime}\right)+p_{z}^{\prime} \cos \theta^{\prime}\right]\right\} \\
& \cdot r^{\prime 2} \sin \theta^{\prime} d \theta^{\prime} d \phi^{\prime} d r^{\prime} .
\end{aligned}
$$

Because of the spherical symmetry, we can perform the $\theta^{\prime}$ and $\phi^{\prime}$ integrations to yield

$$
\begin{aligned}
I_{V}=4 \pi & \int_{0}^{a_{s}} \frac{\sin \left(v r^{\prime}\right)}{v r^{\prime}} r^{\prime 2} d r^{\prime}, \\
& \text { where } v=\left(p_{x}^{2}+p_{y}^{2}+p_{z}^{2}\right)^{1 / 2} .
\end{aligned}
$$


The $r^{\prime}$ integration can be evaluated using integration by parts, and the final result for $I_{V}$ is

$$
\begin{gathered}
I_{V}=V \frac{\sin \left(v a_{s}\right)-v a_{s} \cos \left(v a_{s}\right)}{\left(v a_{s}\right)^{3} / 3}, \\
\text { where } V=4 \pi a_{s}^{3} / 3 .
\end{gathered}
$$

For an electrically small sphere, the arguments of the sine and cosine functions are small, and $I_{V}$ approaches $V$.

The expressions for the cylinder in (32) and the sphere in (35) are in agreement with previous Born approximation results [7], but the notation is different. The validity of the Born approximation has been studied numerically by Kerker et al. [11] for dielectric spheres. They compared plane-wave scattering as given by the Born approximation results in (35) with the exact Mie series [7, ch. 9] for wide ranges of values of $n$ and $\left|k^{\prime}\right| a$ and generated plots of the errors of the Born approximation. Their results show a useful range of values where the Born approximation is within 10 percent of the Mie series results. All of our specific cases for spherical scatterers in Section IV fall within this region where the Born approximation error is less than 10 percent. We have no exact solution for rectangular boxes or cylinders of finite length, but we can expect similar ranges of validity because the shape does not appear in the criteria of validity in (17).

Since we have the scattering matrix for three different shapes, it is possible to derive the scattering matrix for a variety of shapes (such as a cylinder on a box) by superposition. For the box and the cylinder, we have assumed that a flat face is parallel to the $x^{\prime} y^{\prime}$ plane. It is possible to treat arbitrary orientation by introducing appropriate coordinate rotations.

\section{Numerical Results for Plane-Wave Incidence}

The geometry of plane-wave incidence is shown in Fig. 4. The incident plane wave can have either TM (parallel) or TE (perpendicular) polarization. For TM polarization, the incident electric field $\boldsymbol{E}^{i}$ is

where

$$
\boldsymbol{E}^{i}=\boldsymbol{e}_{\theta_{i}} E_{0} \exp \left(i k_{i} \cdot \boldsymbol{r}\right)
$$

$$
\begin{aligned}
\boldsymbol{k}_{i} & =\boldsymbol{K}_{i}+\boldsymbol{e}_{z} k \cos \theta_{i}, \\
\boldsymbol{K}_{i} & =k \sin \theta_{i}\left(\boldsymbol{e}_{x} \cos \phi_{i}+\boldsymbol{e}_{y} \sin \phi_{i}\right),
\end{aligned}
$$

$\theta_{i}$ and $\phi_{i}$ are incident elevation and azimuth angles, $e_{\theta_{i}}$ is a unit vector in the $\theta_{i}$ direction, and $E_{0}$ is a constant. For the incident TM field in (36), the corresponding $b_{1}$ coefficients in (1) are

$$
\begin{aligned}
b_{1}(1, \boldsymbol{K}) & =2 \pi E_{0} \cos \theta_{i} \delta\left(\boldsymbol{K}-\boldsymbol{K}_{i}\right) \\
\text { and } b_{1}(2, \boldsymbol{K}) & =0 .
\end{aligned}
$$

For TE polarization, the incident electric field is

$$
\boldsymbol{E}^{i}=\boldsymbol{e}_{\phi_{i}} E_{0} e^{i k_{i} \cdot r}
$$

where $\boldsymbol{e}_{\phi_{i}}$ is a unit vector in the $\phi_{i}$ direction. For the incident TE field in (38), the corresponding $b_{1}$ coefficients

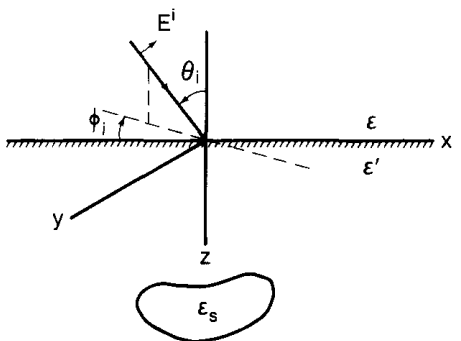

Fig. 4. Geometry for plane-wave incidence.

in (1) are

$$
\begin{aligned}
b_{1}(1, \boldsymbol{K}) & =0 \\
\text { and } b_{1}(2, \boldsymbol{K}) & =2 \pi E_{0} \delta\left(\boldsymbol{K}-\boldsymbol{K}_{i}\right) .
\end{aligned}
$$

The second term in (15) represents the field scattered into region 1 from the buried object, and we define it as

$$
\hat{a}_{1}^{s}=\hat{T}_{12}^{t} \hat{S}_{22}^{\prime} \hat{T}_{21}^{t} \hat{b}_{1} \text {. }
$$

In Section A we present numerical results for the scattered far field, and in Section B we present numerical results for the scattered near field. In both sections we show only the field scattered from the buried object and do not include the specular reflection from the planar interface.

\section{A. Scattered Far Field}

The geometry for the scattered far field is shown in Fig. 5. When $k r$ is large, the scattered field can be evaluated asymptotically $[6$, p. 58]

$$
\begin{aligned}
\boldsymbol{E}_{1}^{s} \sim & i k|\cos \theta| \frac{e^{i k r}}{r} \\
\cdot & {\left[a_{1}^{s}(1, \boldsymbol{K})(-k / \gamma) \boldsymbol{e}_{\theta}\right.} \\
+ & \left.a_{1}^{s}(2, \boldsymbol{K}) \boldsymbol{e}_{\phi}\right]\left.\right|_{\boldsymbol{K}=k \boldsymbol{K} / r} .
\end{aligned}
$$

For all of the numerical results, we set the permeability equal to the free-space value everywhere. For region 1 we set the permittivity $\epsilon$ equal to the free-space value. For region 2 we set the complex relative permittivity $\epsilon^{\prime} / \epsilon=$ $3.0+i 0.1$, and this value is representative of fairly dry sand [12]. For the buried object we set the complex relative permittivity $\epsilon_{s} / \epsilon=2.9+i 0.05$. This value is representative of some plastics and explosives and satisfies the low-contrast condition in (17). Since the scattered field strength is proportional to $\left|n^{2}-1\right|$, results for other values of $\epsilon_{s}$ can be obtained easily. The azimuthal incidence angle $\phi_{i}$ is zero in all cases.

The normalized scattered field strength for $\phi=0$ as a function of $\theta$ is shown in Figs. 6-10 for various cases. The relevant dimensions are normalized to the free-space wavelength $\lambda(=2 \pi / k)$. For a typical frequency of 600 $\mathrm{MHz}, \lambda=50 \mathrm{~cm}$. In all cases, the buried object has a volume of $0.008 \lambda^{3}$.

The dependence on the burial depth of a cube is shown in Fig. 6 for normal incidence $\left(\theta_{i}=0\right)$. For $d / \lambda=0.1$, 


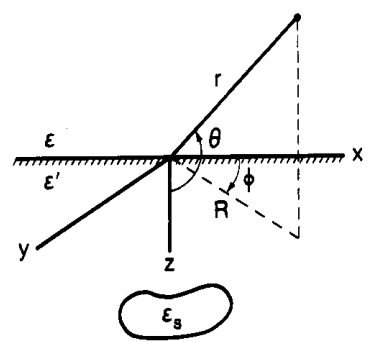

Fig. 5. Geometry for the scattered far-field.

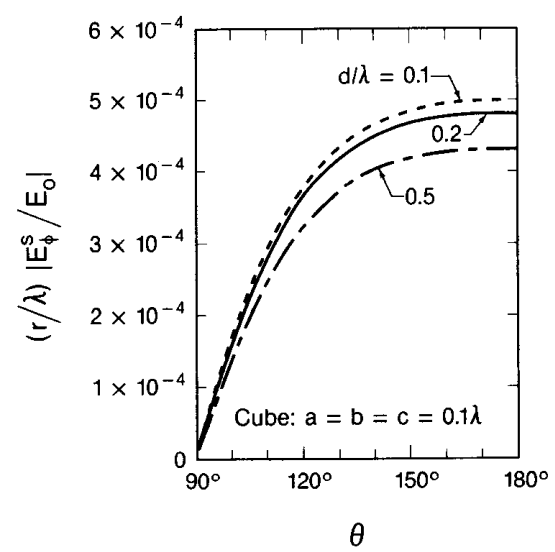

Fig. 6. Scattered far field for a cube located at various depths $d$. Normal incidence $\left(\theta_{i}=0\right)$.

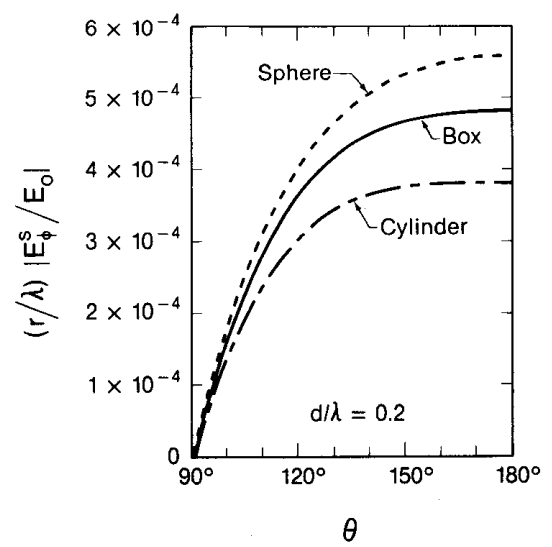

Fig. 7. Scattered far field for a cube $(a=b=c=0.1 \lambda)$, a circular cylinder $\left(h=a_{c}=0.1084 \lambda\right)$, and a sphere $\left(a_{s}=0.1241 \lambda\right)$ for normal incidence. All have equal volumes $\left(V=0.008 \lambda^{3}\right)$.

the upper surface of the cube is flush with the interface. Results for a sphere, a cube, and a cylinder are shown in Fig. 7, and there is some shape dependence. Results for a cube, a flattened box, and an elongated box are shown in Fig. 8. The flattened box has the largest scattered field because of the $\sin \left(p_{z} c\right) /\left(p_{z} c\right)$ factor in (29).

The effect of incidence angle is shown in Figs. 9 and 10. For TE polarization in Fig. 9, the curves lose their

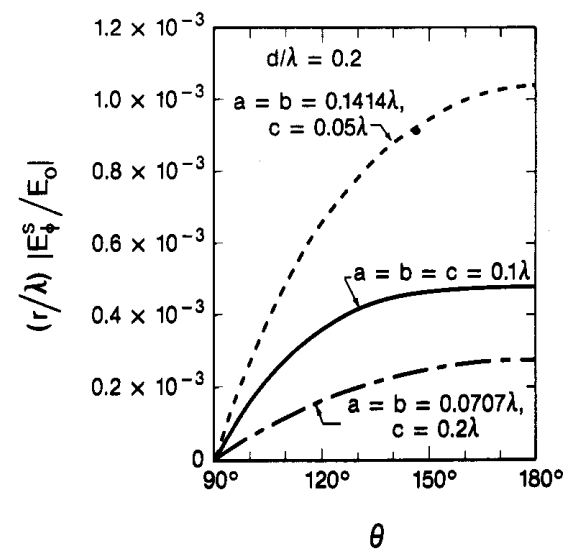

Fig. 8. Scattered far field for equal-volume boxes of various dimensions for normal incidence.

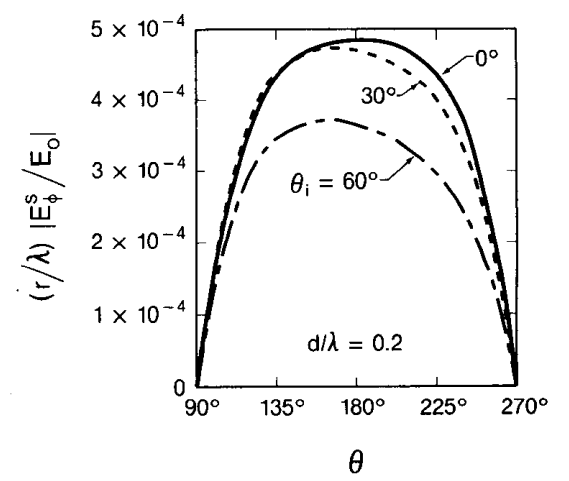

Fig. 9. Scattered far field for a cube for TE incidence at various angles $\theta_{i}$.

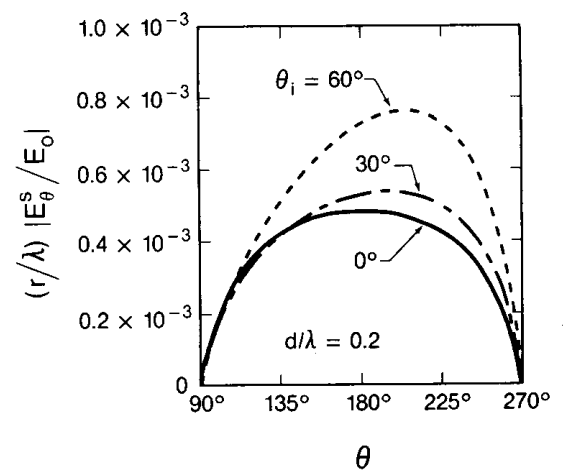

Fig. 10. Scattered far field for a cube for TM incidence at various angles $\theta_{i}$.

symmetry for $\theta_{i} \neq 0$, and the peak is shifted toward the specular direction. However, the general field level decreases because of a decrease in the transmission coefficient $\left(T_{21}\right)$ of the incident field. For TM polarization in Fig. 10, the peaks are shifted in the opposite direction, and this is a result of the polarization properties of the scattering matrix as given by (26). In all cases, the scat- 


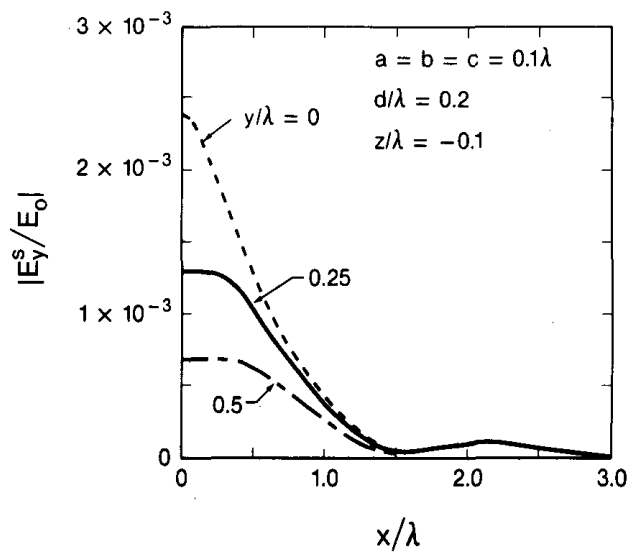

Fig. 11. Scattered near field for a cube for normal incidence.

tered far field is zero at the interface $\left(\theta=90^{\circ}\right)$, and this is consistent with calculations by other methods [4].

\section{B. Scattered Near Field}

The scattered near field is of interest in detection where the receiving antenna is located near the interface. From (6) and (40), the transverse components of the scattered near field are

$$
\begin{aligned}
\boldsymbol{E}_{1 t}^{s}(r)= & \frac{1}{2 \pi} \int \sum_{m} \boldsymbol{\kappa}_{m} a_{1}^{s}(m, \boldsymbol{K}) \exp (-i \gamma z) \\
& \cdot \exp \left[i\left(k_{x} x+k_{y} y\right)\right] d k_{x} d k_{y} .
\end{aligned}
$$

A similar expression for the $z$ component can be obtained from (1). For a fixed value of $z$, the double integration over $k_{x}$ and $k_{y}$ can be approximated [13] by a two-dimensional fast Fourier transform (2D FFT). Thus, a rectangular grid of field values at discrete values of $x$ and $y$ is computed rapidly by $2 \mathrm{D}$ FFT.

In Figs. 11-16, we show the normalized magnitude of $E_{y}^{s}$ as a function of $x$ for TE polarization. There are also small $x$ and $z$ components, but the $y$ component is dominant. The $y$ component is the one of interest in detection systems when $y$-directed dipoles are used for transmitting and receiving.

The near field for several values of $y$ at a height of $0.1 \lambda$ above the interface is shown in Fig. 11. The peak value occurs directly above the buried cube for normal incidence $\left(\theta_{i}=0\right)$. The scattered field is small compared to the incident field because of the low dielectric contrast. The scattered field is somewhat larger for the flattened box in Fig. 12.

The depth dependence is illustrated in Fig. 13. The response is decreased and stretched out as the depth is increased. The height dependence is shown in Fig. 14, and this is important for liftoff considerations of real detectors. It is most desirable to locate the receiving antenna as close to the interface as possible.

The effect of the incidence angle is shown in Fig. 15 for a rectangular box scatterer. For $\theta_{i} \neq 0$, the peak field

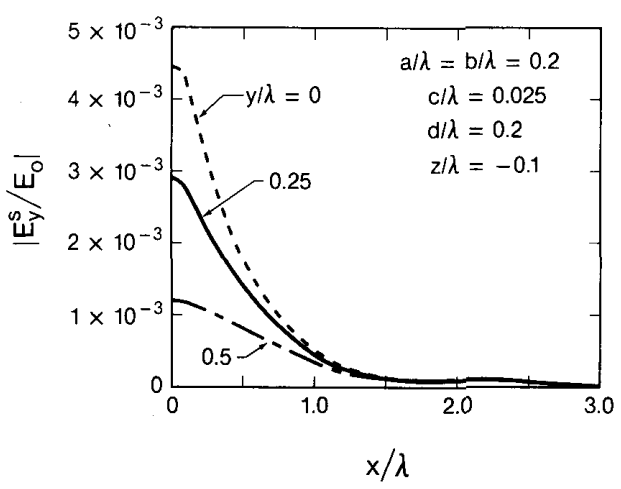

Fig. 12. Scattered near field for a rectangular box for normal incidence.

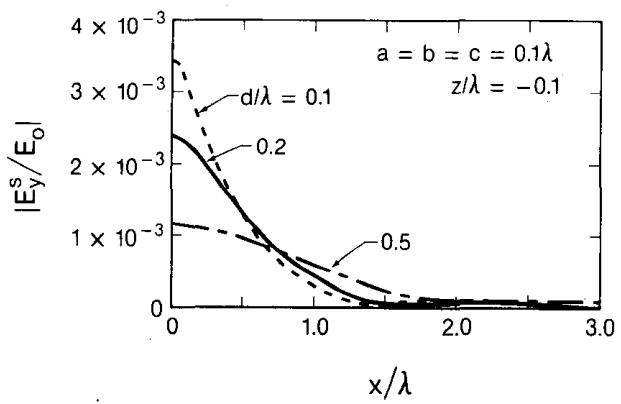

Fig. 13. Scattered near field for a cube located at various depths for norma incidence.

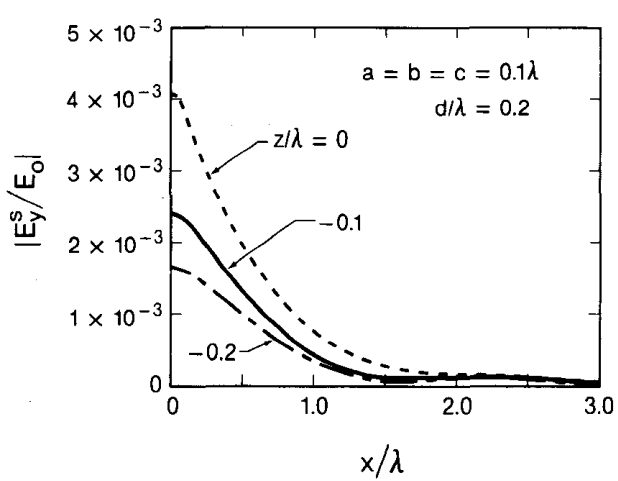

Fig. 14. Scattered near field for a cube at various heights above the interface for normal incidence.

is no longer located directly over the scatterer, but is shifted in the specular direction. This shift in the peak field also occurs for other values of $y$ as shown in Fig. 16.

\section{CONCLUSIONS}

We have used the Born approximation to obtain the scattering matrix for buried objects of low contrast. For general shapes a numerical integration over the volume of the object is required, but for some special shapes (sphere, circular cylinder, and rectangular box) the integration can be done analytically. 


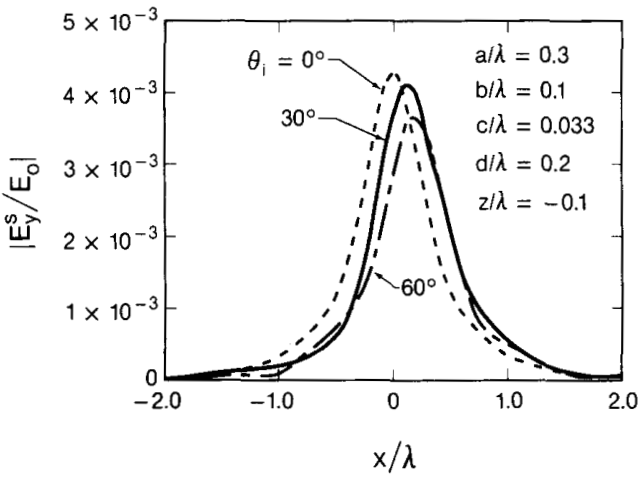

Fig. 15. Scattered near field for a rectangular box for various incidence angles.

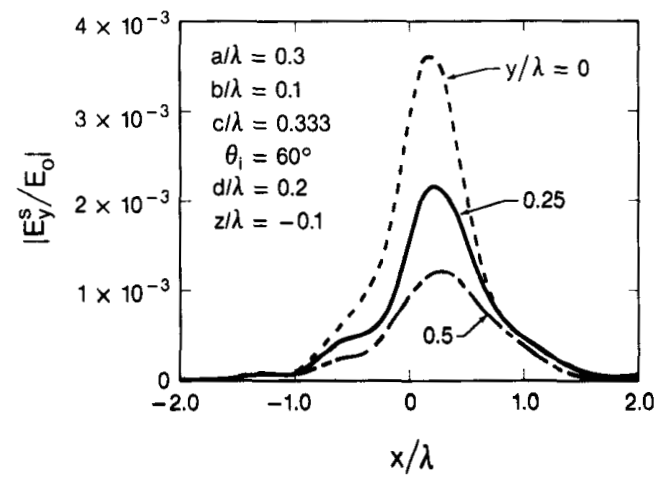

Fig. 16. Scattered near field for a rectangular box for oblique incidence.

We have used the plane-wave scattering-matrix formulation to compute the scattering from buried objects of low contrast. Numerical results have been presented for the scattered far field and near field. Near fields are calculated using 2D FFT, and no Sommerfeld integral evaluations are required. The scattered fields are fairly weak because of the low dielectric contrast, but this is an important case in detection of buried objects.

A number of extensions to this work would be useful. Rather than plane-wave incidence, we could treat the incident field from the transmitting antenna of an actual detector by using the plane-wave scattering matrix theory [5]. The plane-wave characteristics of the receiving antenna could also be included in the calculations. This would allow comparison with UHF detection measurements for dielectric objects buried in sand. Other object shapes could be analyzed by combining the simple shapes studied in this paper. It would also be interesting to carry this approach over to the time domain [14].

\section{APPENDIX A}

SCALAR Form of $S_{22}^{\prime}$

We first rewrite the dyadic scattering matrix of (26) in the following form:

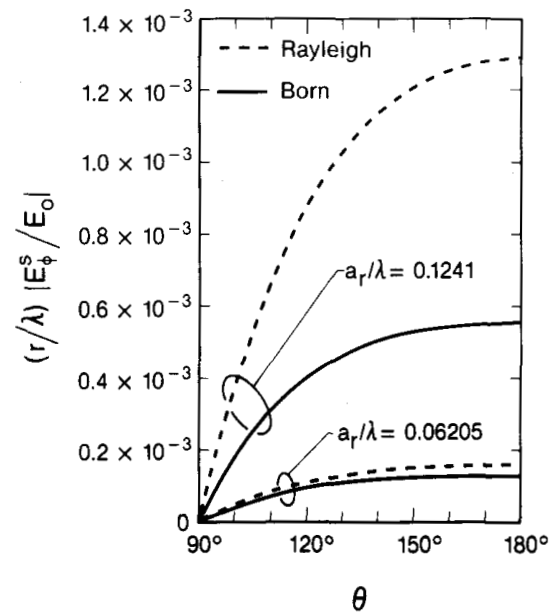

Fig. 17. Comparison of Rayleigh and Born approximations for far field scattered by a sphere at depth $d=0.2 \lambda$, for normal incidence.

$$
\begin{aligned}
\boldsymbol{s}_{22}^{\prime}(\boldsymbol{K}, \boldsymbol{L})=f_{22} \pi\left(\boldsymbol{k}^{\prime-}\right) \cdot \pi\left(\boldsymbol{l}^{+}\right) \\
\text {where } f_{22}=\frac{i k^{\prime 2}\left(n^{2}-1\right) I_{V}}{8 \pi^{2} \gamma^{\prime}} .
\end{aligned}
$$

The conversion from $s_{22}^{\prime}$ to $S_{22}^{\prime}$ is given by Kerns [6], and in general it involves several dot products of vectors and dyads. However, for the special form of $s_{22}^{\prime}$ in (A1), $S_{22}^{\prime}$ reduces to

$$
\begin{aligned}
S_{22}^{\prime}(1, \boldsymbol{K} ; 1, \boldsymbol{L}) & =\frac{k_{z}^{\prime}}{l_{z}^{\prime}} \boldsymbol{e}_{\mathrm{par}}\left(\boldsymbol{k}^{\prime^{-}}\right) \cdot \boldsymbol{e}\left(\boldsymbol{l}^{+}\right) f_{22} \\
S_{22}^{\prime}(1, \boldsymbol{K} ; 2, \boldsymbol{L}) & =\frac{k_{z}^{\prime}}{k^{\prime}} \boldsymbol{e}_{\mathrm{par}}\left(\boldsymbol{k}^{\prime-}\right) \cdot \boldsymbol{\kappa}_{2}(\boldsymbol{L}) f_{22} \\
S_{22}^{\prime}(2, \boldsymbol{K} ; 1, \boldsymbol{L}) & =\frac{k^{\prime}}{l_{z}^{\prime}} \boldsymbol{\kappa}_{2}(\boldsymbol{K}) \cdot \boldsymbol{e}_{\mathrm{par}}\left(\boldsymbol{l}^{\prime^{+}}\right) f_{22} \\
\text { and } S_{22}^{\prime}(2, \boldsymbol{K} ; 2, \boldsymbol{L}) & =\boldsymbol{\kappa}_{2}(\boldsymbol{K}) \cdot \boldsymbol{\kappa}(\boldsymbol{L}) f_{22} .
\end{aligned}
$$

The parallel unit vectors in (A2) are defined as [6]

$e_{\mathrm{par}}\left(k^{\prime-}\right)=\kappa_{2}(K) x \frac{k^{\prime-}}{k^{\prime}}$ and $e_{\mathrm{par}}\left(l^{\prime+}\right)=\kappa_{2}(L) x \frac{l^{\prime^{+}}}{l^{\prime}}$.

\section{Appendix B}

COMPARISON WITH RAYLEIGH SCATTERING FOR A SPHERE

In Rayleigh scattering [7, Sec. 6.1], the electrical size of the scatterer is small compared to the wavelength in the surrounding medium $\left(\left|k^{\prime}\right| a_{s} \ll 1\right)$. Under this condition, the dyadic scattering matrix for a dielectric sphere is $[6, p .130]$

$$
\begin{aligned}
& s_{22}^{\prime}(\boldsymbol{K}, \boldsymbol{L})=f_{22 R} \pi\left(\boldsymbol{k}^{\prime-}\right) \cdot \pi\left(\boldsymbol{l}^{++}\right), \\
& \text {where } f_{22 R}=\frac{3 i k^{\prime 2}\left(n^{2}-1\right) V}{8 \pi^{2} \gamma^{\prime}\left(n^{2}+2\right)} .
\end{aligned}
$$


Comparing (A1) and (B1), we see that the dyadic function is the same, but the scalar functions are slightly different. The ratio of the scalar functions is

$$
f_{22 R} / f_{22}=\frac{3 V}{\left(n^{2}+2\right) I_{V}} .
$$

For low contrast, $n^{2} \approx 1$, and for small $\left|k^{\prime}\right| a_{s}, I_{V} \approx V$. Under these conditions, the ratio in (B2) is approximately 1 , and the Rayleigh and Born approximations agree.

A numerical comparison for scattering by a buried sphere is shown in Fig. 17. The permittivities and the depth of the sphere are the same as in Fig. 7. For $a_{s} / \lambda=$ $0.1241,\left|k^{\prime}\right| a_{s} \approx 1.35$, and Rayleigh scattering is not valid. For $a_{s} / \lambda=0.06205,\left|k^{\prime}\right| a_{s} \approx 0.675$, and Rayleigh scattering is nearly valid. For this case, the Born and Rayleigh curves in Fig. 17 are in fairly close agreement.

\section{ACKNOWLEDGMENT}

The author would like to thank W. K. Klemperer and R. G. Geyer for useful discussions.

\section{REFERENCES}

[1] M. Cauterman, J. L. Martin, P. Degauge, and R. Gabillard, "Numerical modelling for electromagnetic remote sensing of inhomogeneities in the ground,"'Proc. IEEE, vol. 67, pp. 1009-1015, 1979.

[2] G. Kristensson, "Electromagnetic scattering from buried inhomogeneities-a general three-dimensional formalism," J. Appl. Phys. vol. 51 , pp. $3486-3500,1980$.

[3] S. F. Mahmoud, S. M. Ali, and J. R. Wait, "Electromagnetic scattering from a buried cylindrical inhomogeneity inside a lossy earth," Radio Sci., vol. 16, pp. 1285-1298, 1981.

[4] H. S. Chang and K. K. Mei, "Scattering of electromagnetic waves by buried and partly buried bodies of revolution," IEEE Trans. Geosci. Remote Sensing, vol. GE-23, pp. 596-605, 1985.

[5] D. A. Hill and K. H. Cavcey, "Coupling between two antennas separated by a planar interface,"' IEEE Trans. Geosci. Remote Sensing, vol. GE-25, pp. 422-431, 1987.
[6] D. M. Kerns, Plane-Wave Scattering-Matrix Theory of Antennas and Antenna-Antenna Interactions. Washington, D.C.: NBS Monograph 162,1981 .

[7] H. C. van de Hulst, Light Scattering by Small Particles. New York: Wiley, 1957.

[8] W. H. Carter, "Inverse scattering in the first Born approximation," Optical Engineering, vol. 23, pp. 204-209, 1984.

[9] R. C. Weaver, U.S. Army Belvoir Research, Development \& Engineering Center, Fort Belvoir, VA, private communication.

[10] M. Abramowitz and I. A. Stegun, Handbook of Mathematical Functions, (Applied Mathematics Series 55). Washington, DC: NBS, 1964.

[11] M. Kerker, W. A. Farone, and E. Matijevic, "Applicability of Rayleigh-Gans scattering to spherical particles," J. Opt. Soc. Amer., vol. 53, pp. 758-759, 1963

[12] A. R. Von Hippel, Dielectric Materials and Applications. New York: Technology Press of M.I.T., 1954.

[13] A. Q. Howard, Jr., "On approximating Fourier integral transforms by their discrete counterparts in certain geophysical applications," IEEE Trans. Antennas Propagat, vol. AP-23, pp. 264-266, 1975.

[14] D. Quak and A.T. de Hoop, "Time domain Born approximation to the far-field scattering of plane electromagnetic waves by a penetrable object,"' Radio Sci., vol. 21, pp. 815-821, 1986.

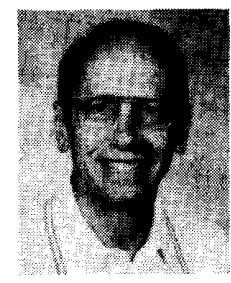

David A. Hill (M'72-SM'76-F'87) was born in Cleveland, $\mathrm{OH}$, on April 21, 1942. He received the B.S.E.E. and M.S.E.E. degrees from Ohio University in 1964 and 1966, respectively, and the $\mathrm{Ph}$.D. degree in electrical engineering from Ohio State University in 1970.

Since 1970 he has been a member of the Boulder scientific community. From 1970 to 1971 he was a Visiting Fellow with the Cooperative Institute for Research in Environmental Sciences where he worked on pulse propagation. From 1971 to 1982 he was with the Institute for Telecommunication Sciences where he worked on theoretical problems in antennas and propagation. Since 1982 he has been in the Electromagnetic Fields Division of the National Bureau of Standards where he has been working on EMC/EMI problems. He is also a Professor Adjoint in the Department of Electrical and Computer Engineering of the University of Colorado.

Dr. Hill is a member of URSI Commissions B, E, and F. He has served as a Technical Editor for the IEEE TRANSACTIONS ON GEOSCIENCE AND REMOTE SENSING and is now an Associate Editor for the IEEE TRANSACTIONS on ANTENNAS AND Propagation. 